\title{
Cubierta para un recinto multifuncional abierto con cuatro paraboloides hiperbólicos textiles
}

\author{
Roofing a multifunctional outdoor court with four \\ hypar fabric structure
}

F. Escrig(*), J. Sánchez-Sánchez ${ }^{(*)}$, M. Ponce ${ }^{(*)}$

RESUMEN

El diseño se refiere a la cubierta de una pista polideportiva para usos multifuncionales con una superficie disponible de $46 \times 24 \mathrm{~m}$. La propuesta consiste en cubrir esta superficie con una superficie textil tensada en una estructura metálica que enmarca cuatro paraboloides hiperbólicos. Lo singular de la propuesta estriba en que los cuatro soportes del conjunto no están situados en la esquina sino en los puntos medios de los lados. Entonces tenemos cuatro alas en voladizo con $23 \mathrm{~m}$ y $12 \mathrm{~m}$ respectivamente. En este artículo también introducimos precedentes y otras aplicaciones.

\section{SUMMARY}

The design corresponds to a roof for a multifunctional sport court with a disposable space area of $46 \times 24 \mathrm{sqm}$. We decided to cover this area with a textile fabric geometrically defined by four hypar surfaces squared into a steel structure. The particular of this design is that the four supports are placed in the middle of the edges and not in the corners as is habitual. Then the structure is a four wing roof with $23 \mathrm{~m}$ of cantilever in the larger direction and $12 \mathrm{~m}$ in the shorter. We introduce also precedents of this solution from other applications.
Palabras clave: Cubiertas textiles; mallas de barras; estructuras de acero; voladizos.
Keywords: Textile fabric roofs; grids; steel structures; cantilevers. 
1. Composición de cuatro paraboloides hiperbólicos equiláteros sobre cuatro soportes en los puntos medios de los lados.

2. Iglesia de Nuestra señora de de México por Félix Candela.

(Foto cedida por el autor).

3. Propuesta para cubrir dos pistas de pádel con cuatro paraboloides hiperbólicos en voladizo y una altura comprendida entre 6 y $10 \mathrm{~m}$.

4. Propuesta para cubrir dos pistas de pádel y un graderío con cuatro paraboloides hiperbólicos. la Divina Providencia en Ciudad

\section{INTRODUCCIÓN}

Las composiciones de paraboloides hiperbólicos equiláteros delimitados por bordes rectos es muy apropiada para resolver aplicaciones estructurales por su facilidad constructiva y su buena apariencia tanto formal como funcional. Entre las muchas posibilidades geométricas que ofrece el sistema elegimos las dos representadas en la Figura 1.

El arquitecto Félix Candela utilizó este sistema en numerosas ocasiones en composiciones a veces muy llamativas. Pero también en las formas simples que nosotros vamos a aplicar a nuestro diseño como se puede ver en la Figura 2 que representa la cubierta de la Iglesia del Refugio en México.

Nosotros hemos sustituido la viga de borde que aparece en todas estas estructuras de hormigón por un marco de acero estabili-
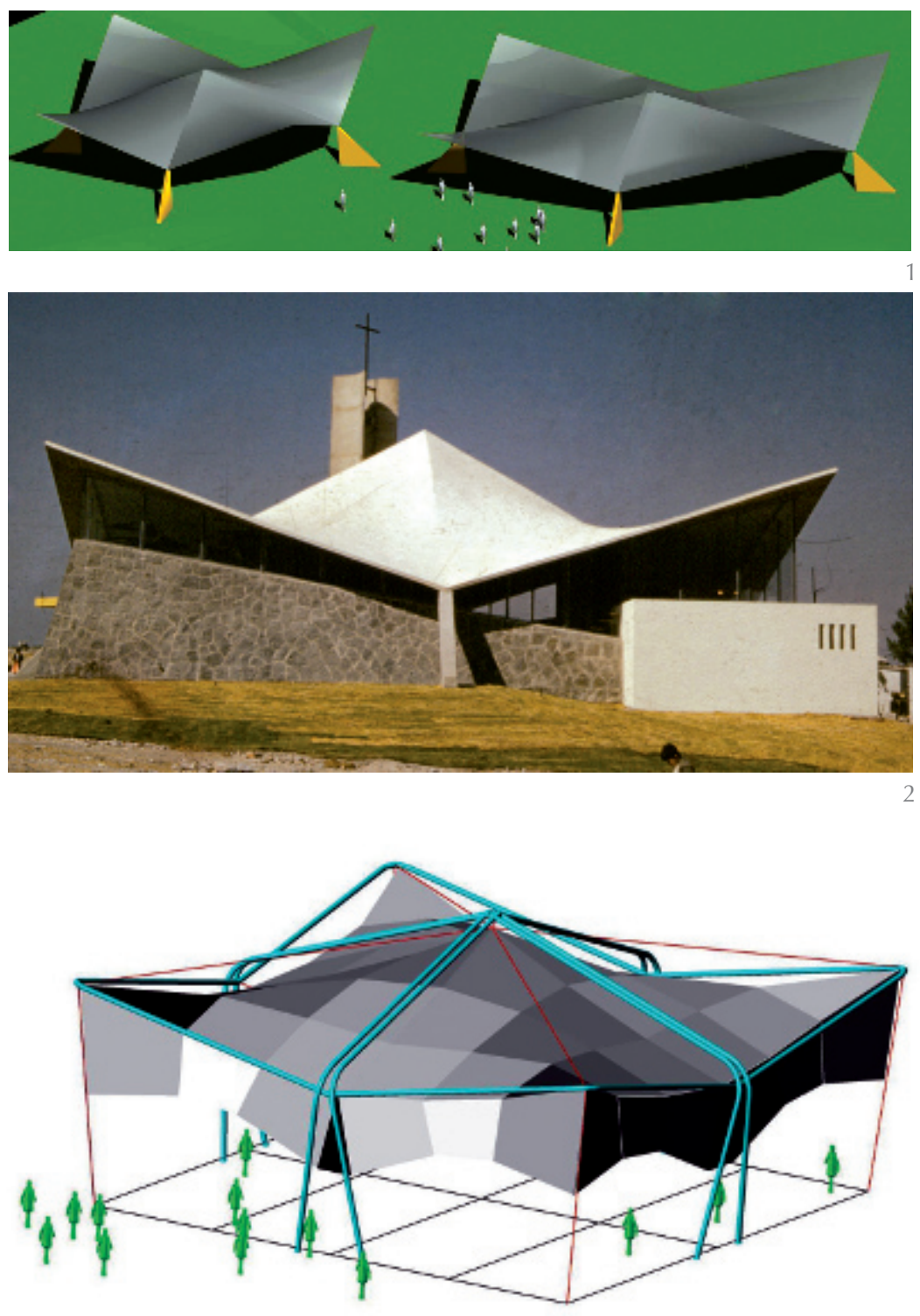

zado con cables como el que se muestra en la Figura 3. En la aplicación que se muestra, con unas dimensiones de $20 \times 20 \mathrm{~m}$ tratamos de proporcionar una solución económica para cubrir dos pistas de pádel.

Si lo que buscamos es una forma rectangular, por ejemplo para la solución anterior pero incluyendo un graderío interior, necesitaríamos ampliar la superficie a $30 \times 20 \mathrm{~m}$ para lo cual necesitamos reforzar los soportes con respecto al caso anterior (Figura 4).

\section{UN NUEVO PABELLÓN PARA USOS MULTIFUNCIONALES}

Las propuestas anteriores no fueron construidas pero se aportan porque fueron la base de un encargo posterior que las desarrollaba a mayor escala. En este caso se trataba de cubrir una pista de $40 \times 20 \mathrm{~m}$ y las soluciones propuestas se sintetizan en la mostrada Figura 5, en donde se han reforzado las vigas interiores y se han estabilizado los soportes laterales, dotando al conjunto de mayor rigidez que los proyectados con anterioridad. Nuestro equipo ya había alcanzado gran experiencia en este tipo de proyectos como se aprecia en la bibliografía (1) (2) (3) (4) (5) (6).

En principio se pensó en rodear las dimensiones de la pista de una banda de 1 $\mathrm{m}$ como playa de la misma, pero luego se vio que resultaba insuficiente y que el área real a cubrir debía ser de $46 \times 24$ m lo que cambiaba sustancialmente la dimensión del problema. Después de algunos análisis tentativos no era posible mantener la geometría propuesta hasta el momento para las nuevas condiciones y era necesario reforzarla con elementos adicionales.

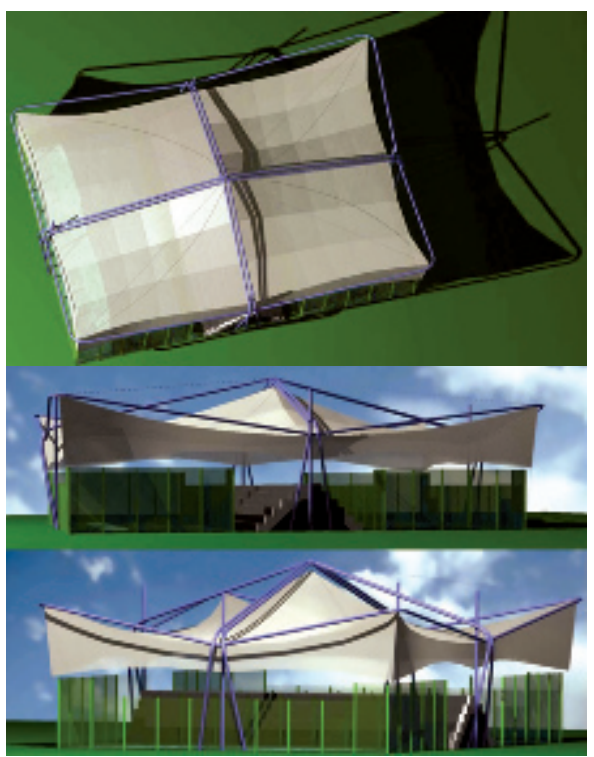



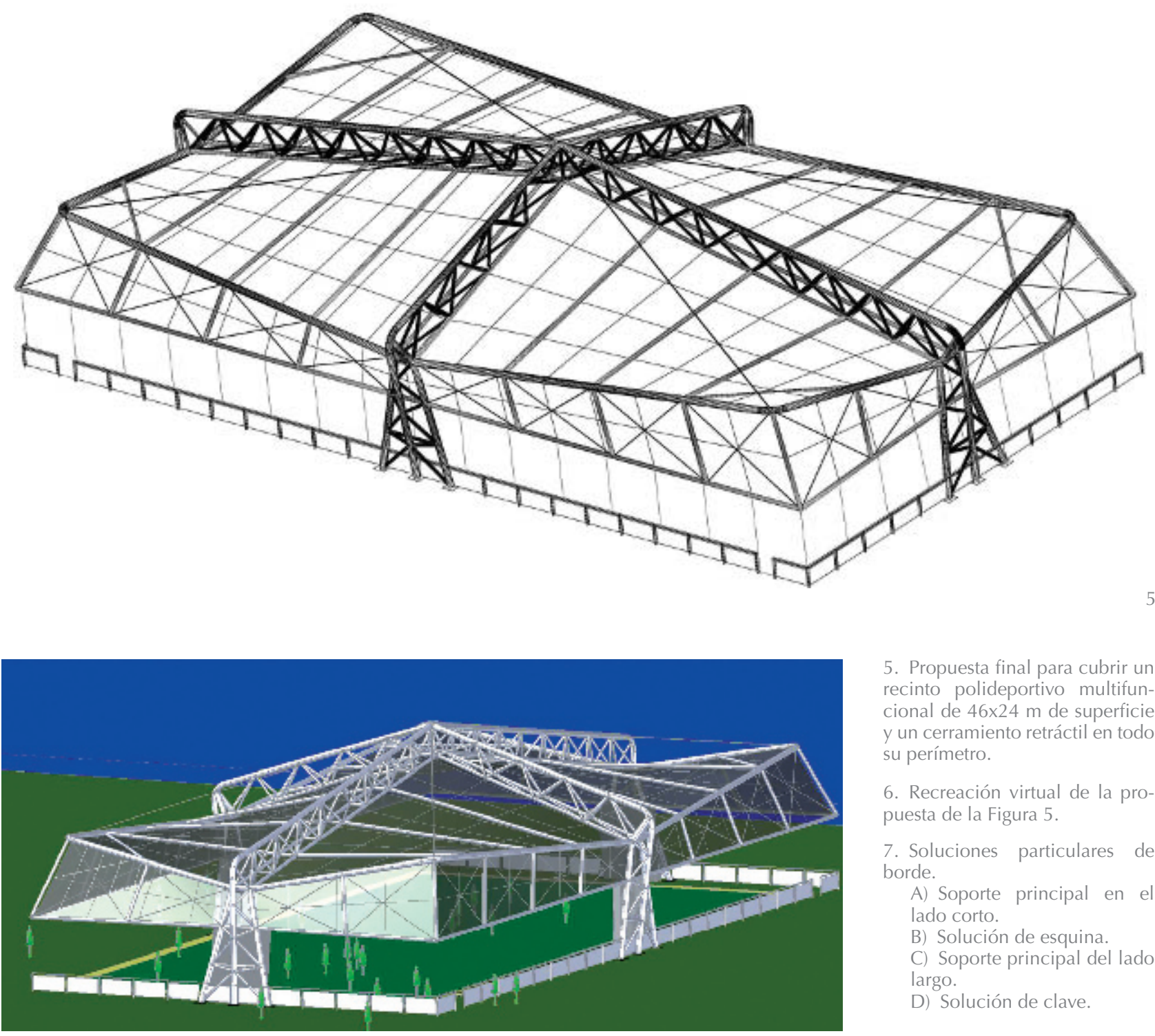

5. Propuesta final para cubrir un recinto polideportivo multifuncional de 46×24 m de superficie y un cerramiento retráctil en todo su perímetro.

6. Recreación virtual de la propuesta de la Figura 5.

7. Soluciones particulares de borde.

A) Soporte principal en el lado corto.

B) Solución de esquina.

C) Soporte principal del lado

largo.

D) Solución de clave.

Por otra parte se añadió otra petición a la del encargo de cubierta. Ahora se trataba de hacer un cerramiento retráctil, por lo que necesitábamos una viga horizontal de rodadura que debíamos incluir en el diseño. También había que considerar la necesidad de evacuar las aguas por un punto bajo que drenara fuera de la pista. Se planteó entonces la solución que se muestra en la Figura 6 que resultó ser ya la propuesta definitiva.

Para resolver constructivamente el proyecto se aportaron las disposiciones constructivas capaces de dar solución a todos los aspectos que consolidaran su funcionamiento. En la Figura 7 se detallan los encuentros en los bordes, en las esquinas y en la clave. Nótese que los soportes de los lados cortos no son iguales que los de los lados largos puesto que tienen intercambiada la inclinación de la pieza central con respecto a las laterales. Ello era debido a la necesidad

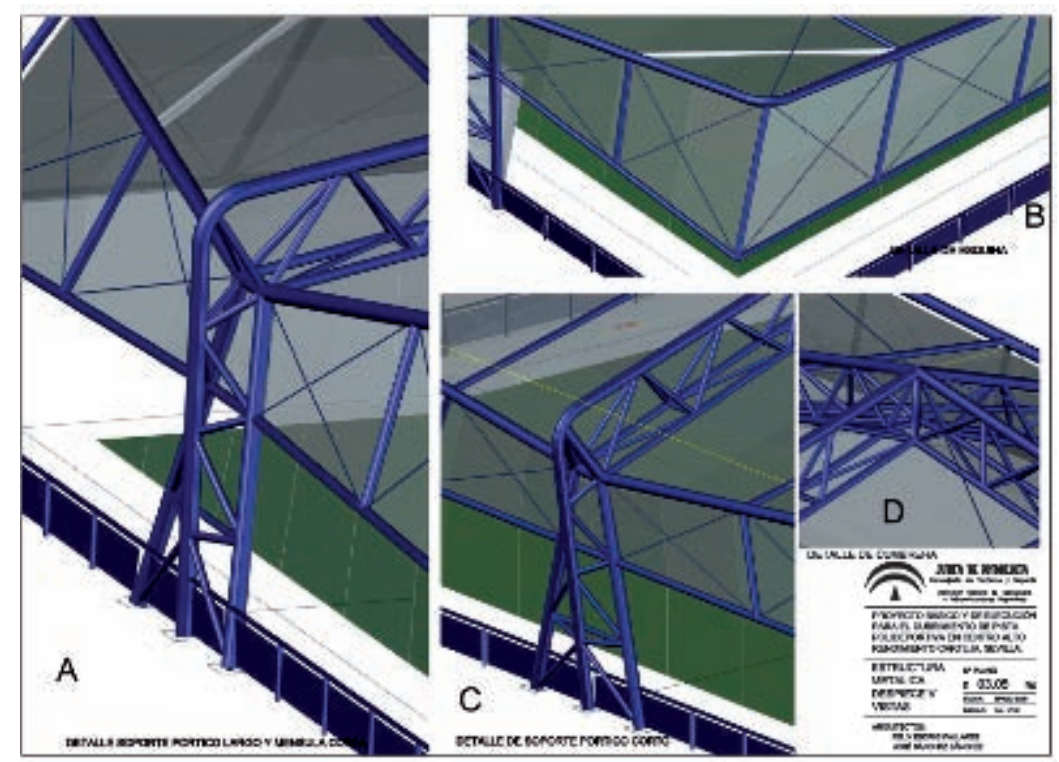



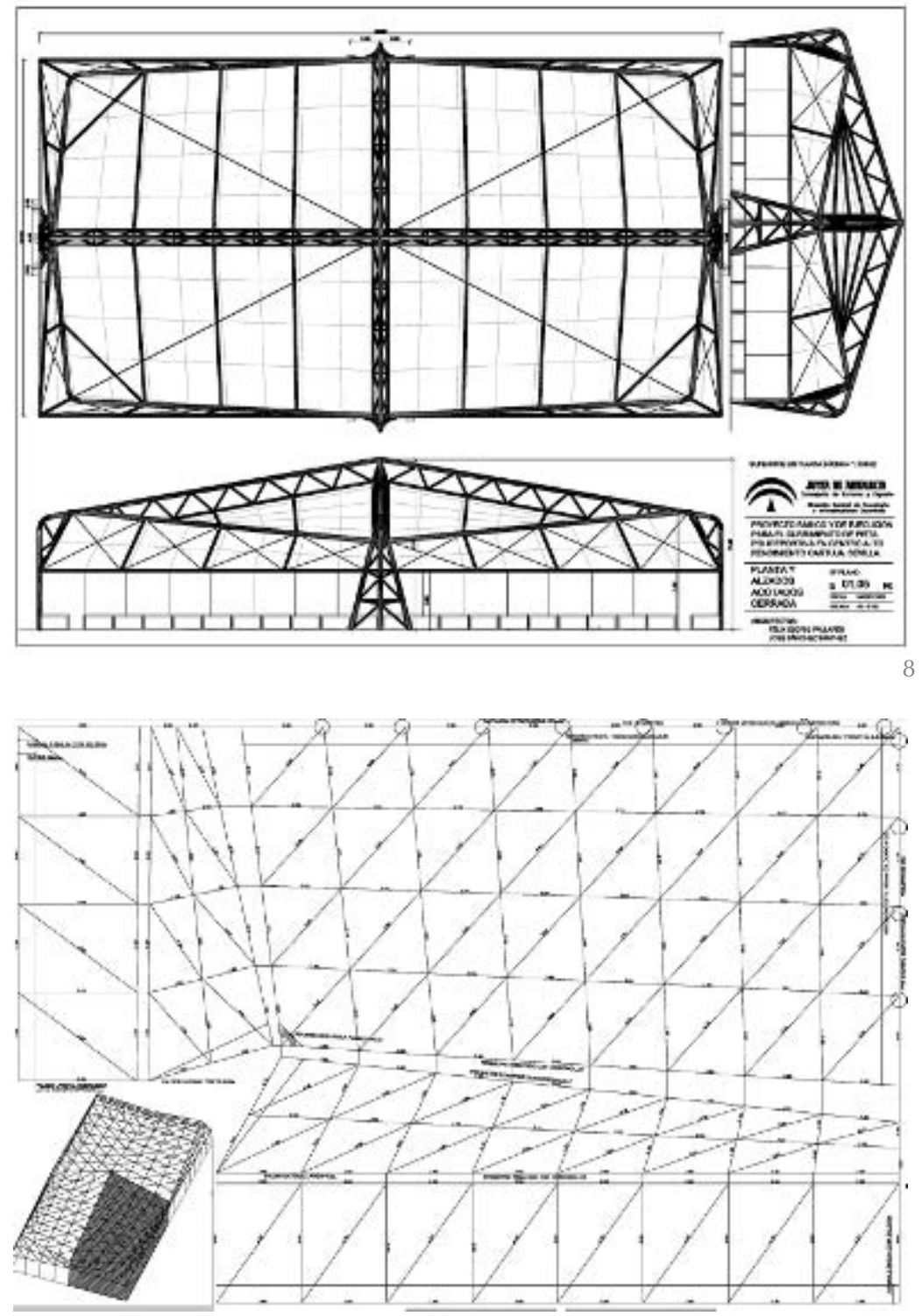

9

8. Plantas y alzados de la cubierta en donde se aprecia que la solución no es estrictamente rectangular.

9. Dimensiones de los patrones de corte de la cubierta textil y posicionamiento de los mismos, incluyendo el cerramiento lateral deslizante.

10. Detalles constructivos de la cogida del textil a la estructura de acero. de minimizar la luz a salvar con las vigas centrales, lo que además produce un descuadre en la planta como se muestra en la Figura 8.

En la Figura 9 se muestra el cerramiento textil, con función estructural puesto que tiene que resistirse a sí mismo y a las sobrecargas de todo el conjunto para transmitirlas a las vigas centrales y los bordes al igual que haría una superficie rígida en paraboloide hiperbólico de hormigón.

El conjunto se dotó además de elementos adicionales importantes para su funcionamiento, como cables estabilizadores de los recuadros de la estructura lateral, sistema de ventilación en cumbrera para evitar condensaciones en el interior de la superficie de cubierta y proporcionar un flujo de aire permanente, a pesar de que este estaba garantizado por ser una cubierta muy ventilada, y un cerramiento de trama abierta deslizante sobre guías, de tal modo que fácilmente puede ser retirado o extendido.

El cálculo se realizó por medio del programa de elementos finitos SAP2000 y resultó un dimensionamiento consistente en tubos de $\varnothing 323,8$ para los elementos principales y de $\varnothing 120,5$ para los demás. Las acciones de la cubierta textil se incorporaron a la estructura tubular y fueron obtenidas de las fuerzas de membrana de los paraboloides hiperbólicos sobre los bordes. Todas las piezas fueron prefabricadas en taller y transportadas a obra para ser ensambladas con las mínimas soldaduras posibles.

Como se aprecia en la Figura 8 fue necesario introducir tres codales que partieran la superficie para evitar la flexión lateral de

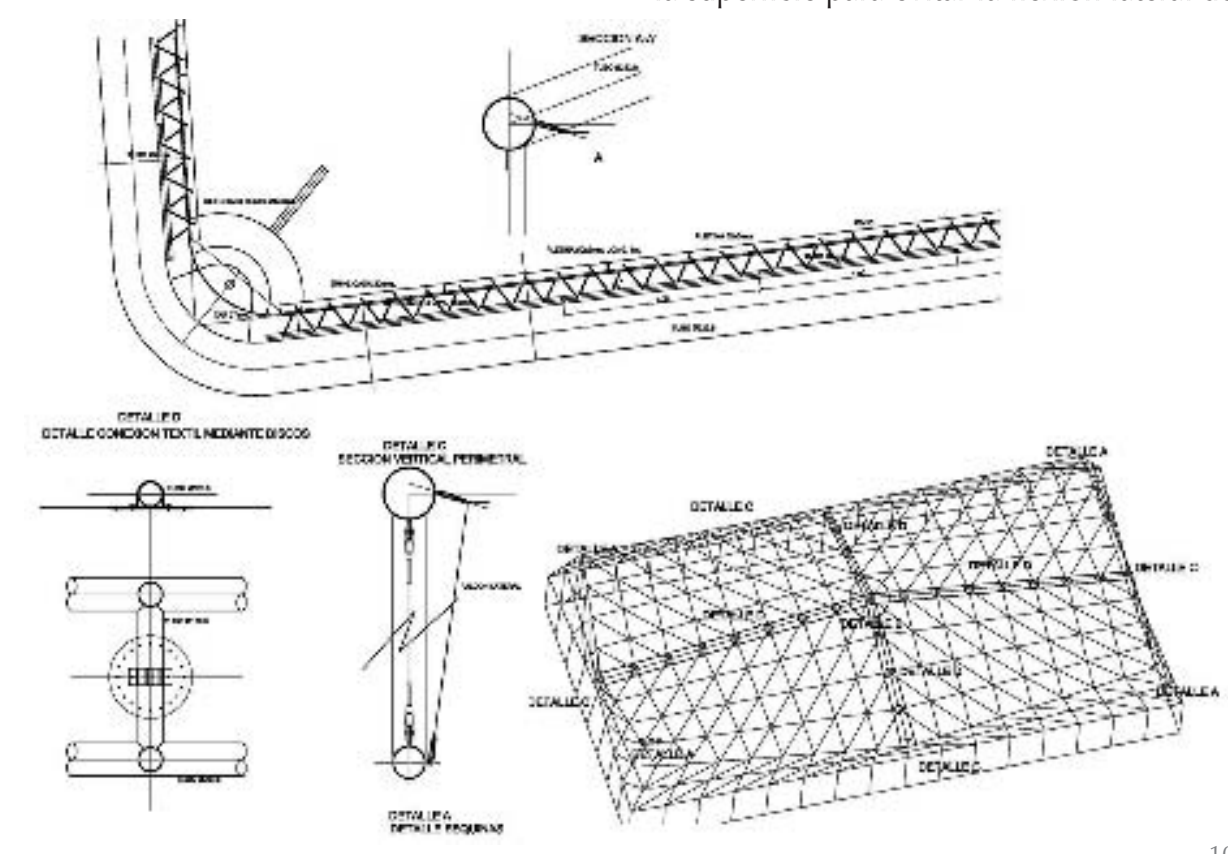


las cerchas de borde y unos codales a $45^{\circ}$ en las esquinas. Ninguno de estos elementos toca a la superficie textil.

En la Figura 10 mostramos algunos de los detalles constructivos de las solución utilizada para cada elemento.

En las Figuras 11 y 12 se muestran algunos aspectos de la fabricación y transporte, que requirió medios especiales.

\section{PROCESO CONSTRUCTIVO}

En obra la instalación fue sencilla puesto que el control de la geometría se había seguido escrupulosamente, así como todas las precauciones de posicionamiento (Figuras 13 y 14).

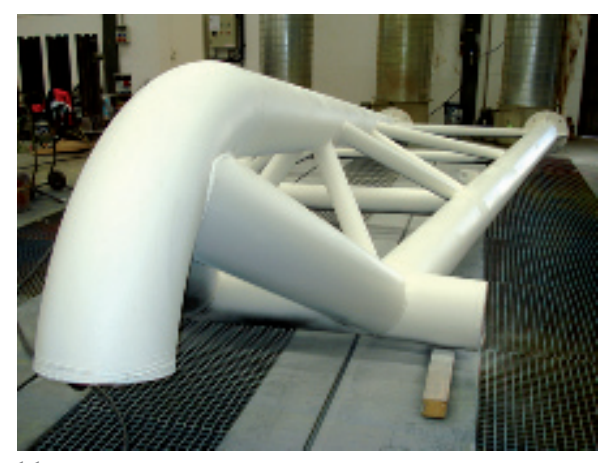

En la Figura 15 podemos ver los particulares de la solución del complicado nudo de esquina en donde confluyen la cubierta, los laterales y el puño, cada elemento con su sistema de tensado. Se aprecia que el textil de la cubierta se ha tensado mediante cordón vinculado a un tubo lateral de $\varnothing 20$ soldado a la estructura principal cada metro. Y en la Figura 16 la solución de encuentro con los soportes laterales.

\section{CUESTIONES FUNCIONALES}

Una vez terminada la instalación el conjunto adquirió el aspecto que se muestra en las Figura 17, visto desde el exterior y Figura 18, visto desde el interior.

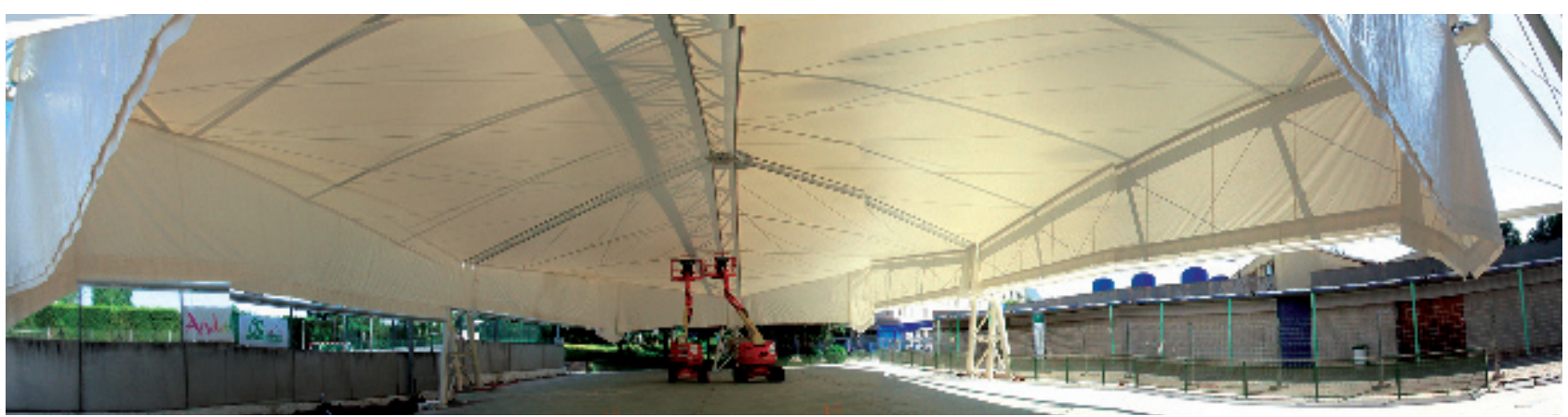

De la experiencia de la utilización posterior de esta instalación sacamos algunas conclusiones interesantes. La primera que el comportamiento térmico era el adecuado puesto que, incluso en los meses de máximo calor, se tenía sensación de confort. La segunda que si orientábamos los focos adecuadamente la iluminación conseguida era sumamente uniforme hasta el punto de no dar sombra, como se aprecia en las Figuras 19 y 20. Por cuestiones presupuestarias las luminarias instaladas fueron las existentes antes de efectuar el cubrimiento, idénticas a las que existen en la pista contigua, que se muestra en la Figura 20, en donde además se advierten las sombras arrojadas.

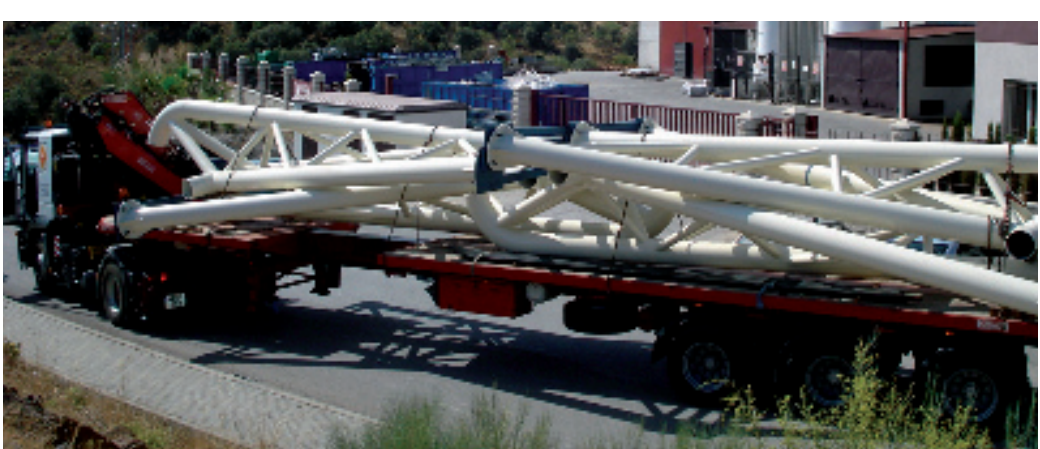

12

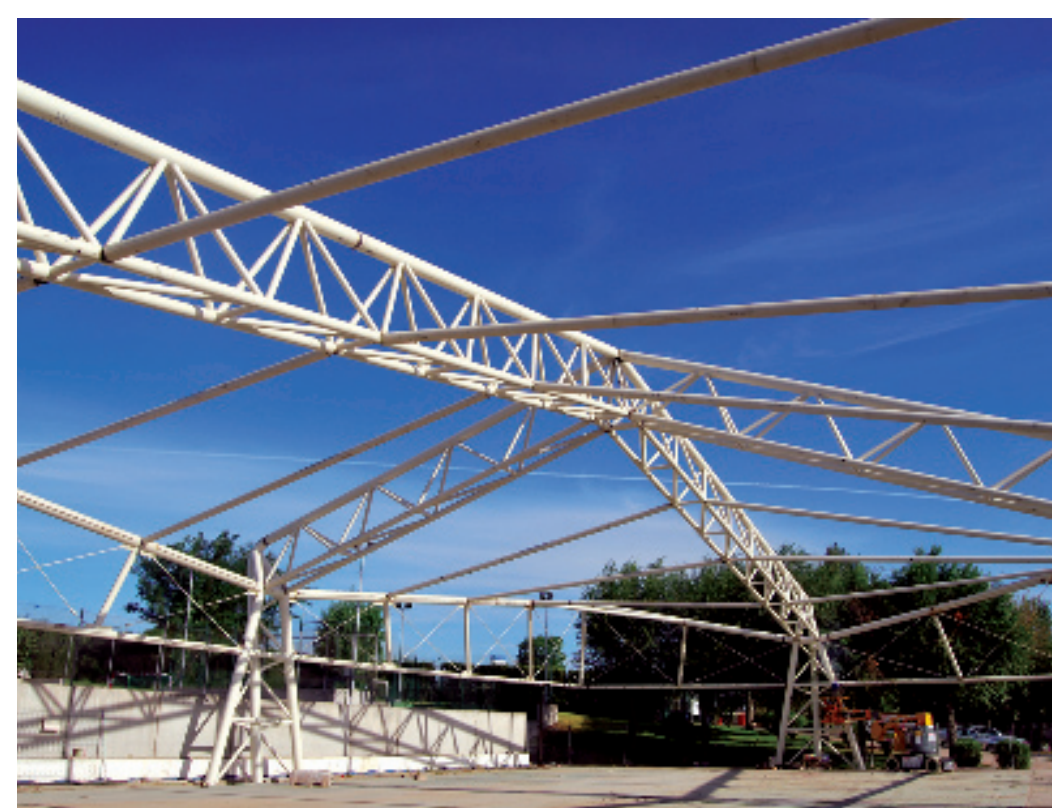

11. Proceso de fabricación y

11. Proceso de fabricación y
pintado en taller de uno de los

2. Transporte a obra de la estructura mediante medios especiales.

\section{Montaje en obra de la es- tructura en donde se aprecian por haberse roto la imprimación, los puntos de soldado in situ. \\ 14. El montaje de la cubierta se realizó en cuatro partes por facilidad constructiva ya que el paquete completo pesaba dema- siado para su manejo.}


15. Solución constructiva de la esquina donde confluye la cubierta, los faldones laterales y el puño.

16. Solución constructiva del encuentro de cubierta y soportes principales. La cubierta pasa limpiamente por delante de la estructura sin tocarla.

17. Vista aérea del conjunto terminado con todos los elementos posicionados y el lateral parcialmente abierto.

18. Vista interior del conjunto terminado con los faldones laterales plegados.

19. Vista nocturna de la pista en donde se aprecia la uniformidad de la distribución lumínica sobre el terreno de juego gracias a la capacidad reflectante de la cubierta.

20. Aspecto exterior nocturno del recinto iluminado.
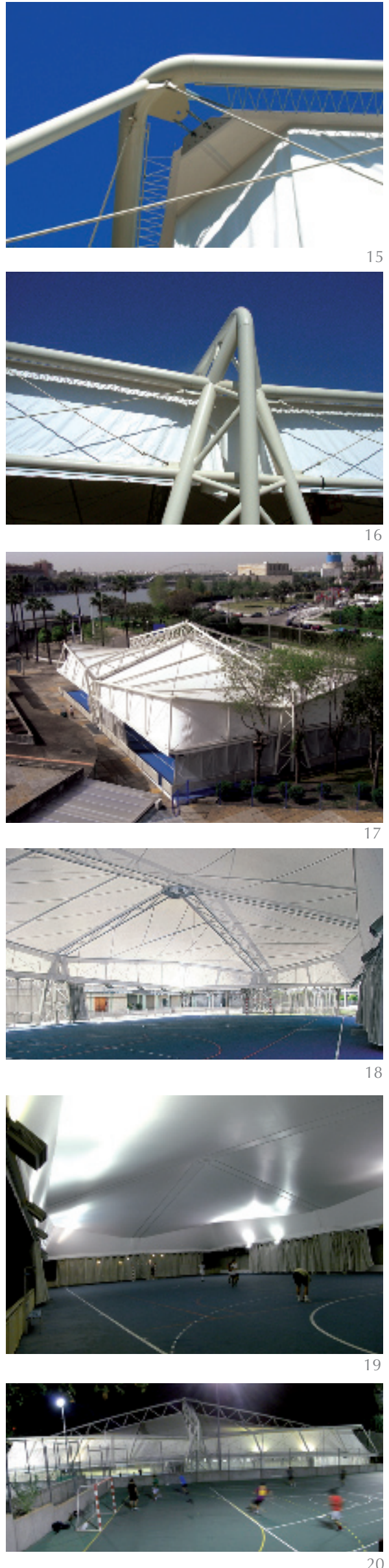

La tercera cuestión que comprobamos fue que, como ya es bien conocido, estas cubiertas no tienen un buen comportamiento acústico. Este aspecto fue chequeado por el equipo de la Escuela de Arquitectura de Sevilla dirigido por el Profesor Teófilo Zamarreño (7) con unas conclusiones parciales que citamos:

En la Figura 21 se presentan los valores medidos y simulados para el tiempo de reverberación (T30), el tiempo de reverberación inicial (EDT), el tiempo central (TS), la definición (D50), la claridad (C80) y la fuerza sonora (G), promediados espacialmente, para las dos posiciones de la fuente, frente a la frecuencia.

El tiempo de reverberación muestra el comportamiento típico para este tipo de recintos: valores muy bajos para la octava inferior asociados con la "transparencia acústica" de la lámina ligera tensionada. Los valores a medias y altas frecuencias, sin embargo, son excesivamente altos para los usos habituales, en los que consideramos fundamental la comunicación verbal. Este efecto se muestra también en los parámetros EDT, Ts y G fundamentalmente. Así mismo, para los valores medidos, observamos un comportamiento que depende de la ubicación de la fuente sonora y que se pone claramente de manifiesto en los valores de EDT, Ts e incluso, en los de T30. Cuando la fuente se sitúa en el centro de la pista el campo reverberado presenta un comportamiento más uniforme, como pone de manifiesto la valoración de la distribución espacial. Los valores simulados sin embargo no muestran este comportamiento con tanta claridad y se aproximan bien, en general, a los medidos con la fuente en F2. Conviene señalar que los valores simulados de T30 se sitúan entre las curvas correspondientes a las dos posiciones de la fuente, ya que en la calibración se utilizaron los valores promedio correspondientes a ambas posiciones.

Las medidas realizadas in situ han puesto de manifiesto que su comportamiento acústico es típico de espacios cubiertos con estructuras textiles tensionadas: con valores de $T$ muy pequeños a bajas frecuencias $y$ excesivamente altos a medias y altas, resultando acústicamente no adecuado para los usos a los que se destina.

Aunque las conclusiones de este estudio eran demoledoras, cosa que ya sabíamos debemos decir a favor de este tipo de soluciones que el sonido directo en actividades deportivas es tan alto en comparación con el reflejado que los estudios anteriores resultan sólo de tipo teórico. Por otra parte si se utiliza 

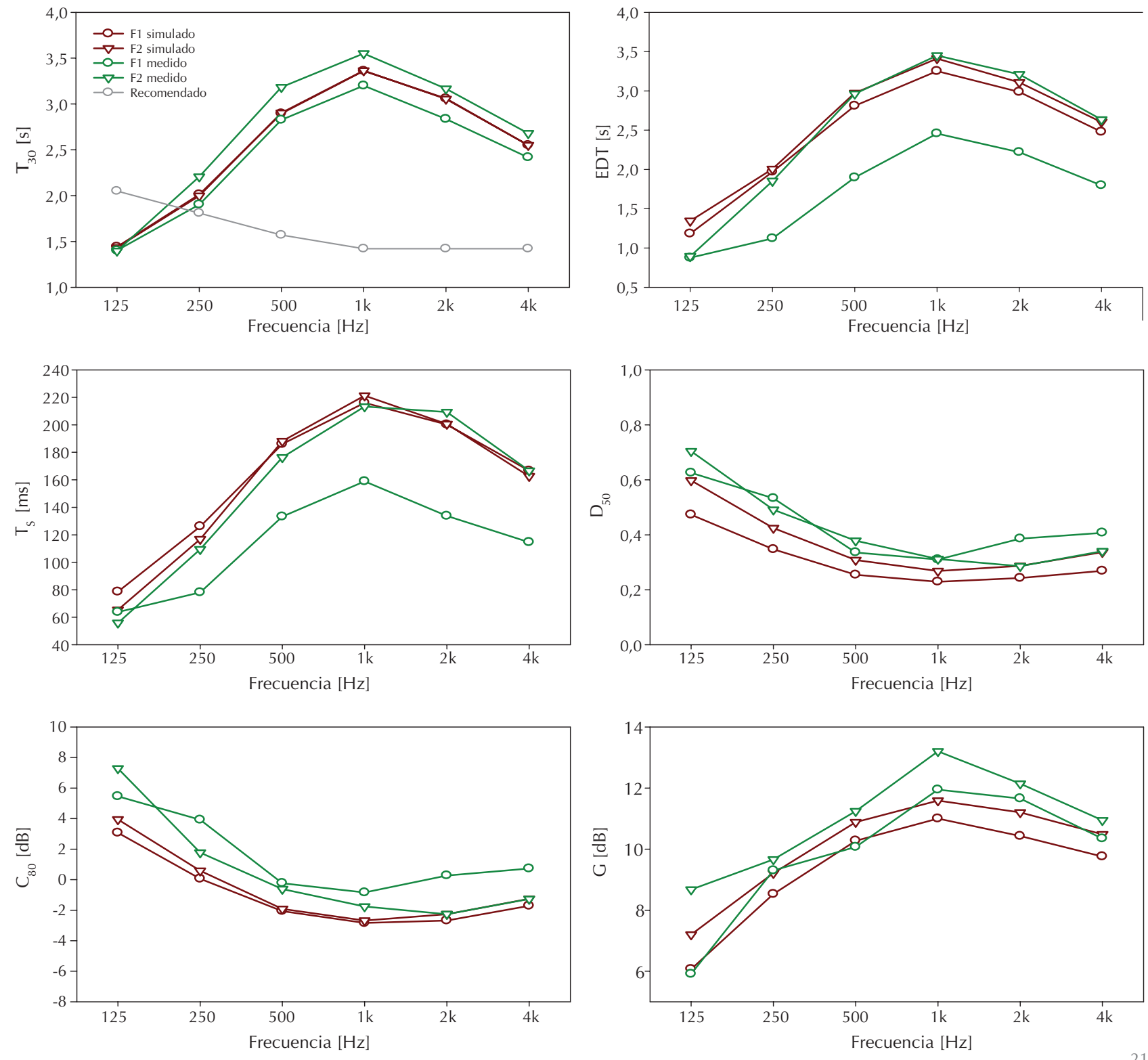

el recinto para actividades de tipo acústico tales como conciertos y mítines el efecto absorbente del público de la sala cambia totalmente los parámetros citados en el informe.

\section{CONCLUSIONES}

El cubrimiento parcial de recintos deportivos como el que hemos realizado en la obra descrita resulta sumamente adecuado en climas como el de Sevilla en que la mayor parte del año se presenta seco y soleado. Las ventajas de este tipo de solución son:

- De tipo económico por la sencillez de las propuestas estructurales y la rapidez de montaje.

- De tipo urbanístico puesto que estas instalaciones se catalogan como cubiertas eventuales y por tanto no consolidan volumen edificado.
- De tipo funcional puesto que no necesitan climatización ni ventilación forzada. La iluminación proporciona niveles de gran atractivo y eficiencia y el comportamiento acústico, aún siendo deficiente puede corregirse con determinadas condiciones que en este caso no eran necesarias.

- De tipo formal porque presentan modelos arquitectónicos novedosos y elegantes.

- Para corroborar estos aspectos nos gustaría citar el parecido de esta cubierta con la que al mismo tiempo se estaba construyendo en el Battery Park del río Hudson de Nueva York para el City Ferry de la Figura 22. Aquí se enfrentaban al mismo problema que nosotros y una solución similar resolvía también problemas económicos, urbanísticos, funcionales y formales en un lugar muy delicado de la ciudad. Sin embargo aunque el aspecto 


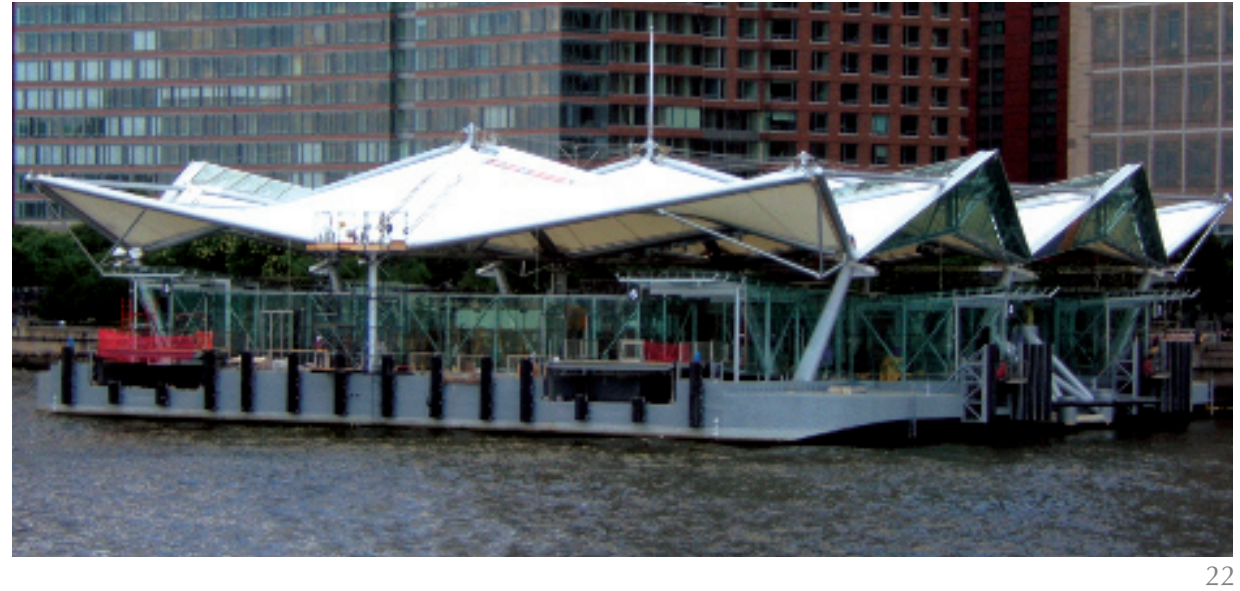

es parecido el sistema estructural difiere en que existen unos tirantes superiores que convierten esta propuesta en una estructura estérea convencional.

\section{CRÉDITOS}

Nombre del proyecto: Cubierta sobre una pista multifuncional en el Centro de Alto Rendimiento de la Cartuja de Sevilla.

Localización: Cartuja de Sevilla

Cliente: Consejería de Turismo y Deportes de la Junta de Andalucía

Función del proyecto: Cubierta de tipo eventual.

Año de construcción: 2010

Arquitectos: Felix Escrig (felix@us.es) y José Sánchez (josess@us.es).
Arquitecto Técnico: Juan Ramón Baeza (jbaeza@us.es)

Ingeniería del conjunto: Performance Ideas y Aplicaciones S.L. performance@arquired.es www.performance-starbooks.com Contratista principal: SANROCON S.A. sanrocon@sanrocon.com

Contratista de la membrana: ARQUITECTURA TEXTIL S.L.

info@arquitecturatextil.com www.arquitecturatextil.com Material: Roof: Naizil Type III www.naizil.it, Wall: VALMEX TF 400 MEHELER www.bric.com Superficie cubierta: $1104 \mathrm{~m}^{2}$

\section{BIBLIOGRAFÍA}

(1) Escrig, F.; Sanchez, J.: "More on Vierendeel Structures to build Great Roofs". Evolution and trends in Design, Analysis and Construction of Shell and Space Structures. IASS Valencia 2009, pp. 1318-1327. ISBN 978-84-8363-461-5.

(2) Escrig, F.; Sánchez, J.; Compan, V.: "Las Vigas Vierendeel Curvas para la Construcción de grandes cubiertas". Hormigón y Acero, Vol. 61 n 255 (2010), pp. 43-53 ISSN 04395689.

(3) Escrig, F.; Sánchez, J.: "La construcción del Velódromo de Dos Hermanas en Sevilla". Informes de la Construcción, Vol. 59 no 508 (2007), pp. 5-27. doi:10.3989/ic.2007. v59.i508.

(4) Escrig, F.; Sánchez, J.: "Proyecto de Cubierta para el Velódromo de Dos Hermanas". Hormigón y Acero. №246 (2007) pp. 5-32 ISSN 0439-5689.

(5) Escrig, F.; Sánchez, J.: "A New Roof Grid to Cover a Large Area: The Vierendeel Solution". International Journal of Space Structures, Vol. 19 n 3 (2004) pp. 177-194. ISSN 0266-3511

(6) Escrig, F.; Sanchez, J.: "Diseño y análisis de una gran cubierta y criterios de diseño". Informes de la Construcción, Vol. 55 n 490 (2004), pp. 49-61. doi:10.3989/ic.2004. v55.i490.

(7) Álvarez, L; Barroso, J.R.; Galindo, M.; Girón, S.; Zamarreño, T.: "Acústica de una pista polideportiva multiusos con cubierta en membrana textil tensionada". 42 Congreso Español de Acústica European Symposium on Environmental Acoustics and on Building Acoustically Sustainable. TecniAcústica. Cáceres 2011. 